\title{
1 Constitutively active SARM1 variants found in ALS patients induce
}

\section{2 neuropathy}

4 A. Joseph Bloom ${ }^{1 *}$, Xianrong Mao ${ }^{1}$, Amy Strickland ${ }^{1}$, Yo Sasaki ${ }^{1}$, Jeffrey Milbrandt ${ }^{*}$, Aaron 5 DiAntonio $^{2 *}$

$7 \quad{ }^{1}$ Needleman Center for Neurometabolism and Axonal Therapeutics and Department of Genetics,

8 Washington University School of Medicine in Saint Louis, St. Louis, MO, USA.

$9 \quad{ }^{2}$ Needleman Center for Neurometabolism and Axonal Therapeutics and Department of

10 Developmental Biology, Washington University School of Medicine in Saint Louis, St. Louis,

11 MO, USA.

$12 *$ corresponding authors

\section{Author email addresses:}

15 A. Joseph Bloom ajbloom@wustl.edu

16 Xianrong Mao_maox@wustl.edu

17 Amy Strickland amy.strickland@wustl.edu

$18 \quad$ Yo Sasaki $\quad$ sasaki@wustl.edu

19 Jeffrey Milbrandt_jmilbrandt@wustl.edu

20 Aaron DiAntonio_diantonio@wustl.edu

23 Running title: SARM1 ALS patient variants 
bioRxiv preprint doi: https://doi.org/10.1101/2021.04.16.439886; this version posted April 16, 2021. The copyright holder for this preprint (which was not certified by peer review) is the author/funder, who has granted bioRxiv a license to display the preprint in perpetuity. It is made available under aCC-BY-NC 4.0 International license. 


\section{Abstract}

2 In response to injury, neurons activate a program of organized axon self-destruction initiated by

3 the NAD ${ }^{+}$hydrolase SARM1. In healthy neurons SARM1 is autoinhibited, but single amino acid

4 changes can abolish autoinhibition leading to constitutively-active SARM1 enzymes that

5 promote degeneration when expressed in cultured neurons. To investigate whether naturally-

6 occurring human variants might similarly disrupt SARM1 autoinhibition and potentially

7 contribute to risk for neurodegenerative disease, we assayed the enzymatic activity of 29 rare

8 SARM1 alleles identified among 8,507 amyotrophic lateral sclerosis (ALS) patients. Ten

9 missense variants or small in-frame deletions exhibit constitutive NADase activity, including

10 more than half of those that are unique to the ALS patients or that occur in multiple patients.

11 Expression of these constitutively active ALS-associated SARM1 alleles in cultured dorsal root

12 ganglion (DRG) neurons is pro-degenerative and cytotoxic. Intrathecal injection of an AAV

13 expressing the common SARM1 reference allele is innocuous to mice, but a construct harboring

$14 S A R M 1^{V 184 G}$, the constitutively active variant found most frequently in the ALS patients, causes

15 axon loss, motor dysfunction, and sustained neuroinflammation. These results implicate rare

16 hypermorphic SARM1 alleles as candidate genetic risk factors for ALS and other

17 neurodegenerative conditions.

19 Keywords: ALS; SARM1; neurodegeneration; axon; NAD

\section{Main Text}

22 Trauma and disease in the nervous system activate an intrinsic axon self-destruction pathway,

23 also known as Wallerian degeneration, which facilitates the orderly clearance of damaged axon 
1 segments. This choice between maintaining or actively dismantling axons is primarily

2 determined by the action of SARM1, a TIR-containing NAD ${ }^{+}$hydrolase that cleaves $\mathrm{NAD}^{+}$to

3 generate nicotinamide and cyclic ADPR (cADPR), a useful biomarker of SARM1 activity ${ }^{1}$. In

4 healthy neurons, SARM1 is maintained in an autoinhibited state, but injury- or disease-induced

5 depletion of the axon survival factor NMNAT2 activates SARM1 leading to a rapid loss of

$6 \mathrm{NAD}^{+}$, metabolic catastrophe, and axon fragmentation ${ }^{2-4}$. SARM1 knockout mice are viable and

7 without apparent phenotypes under routine conditions, but are protected against

8 neurodegeneration in models of axotomy, traumatic brain injury, peripheral neuropathy,

9 glaucoma, and retinal degenerative diseases ${ }^{5-11}$. Conversely, mutations that decrease NMNAT2

10 activity lead to polyneuropathy in both humans and model organisms ${ }^{12,13}$, suggesting that

11 aberrant SARM1 activation has a role in human disease. Furthermore, the recent observation that

12 single point mutations in SARMI can disrupt enzyme autoinhibition ${ }^{14-18}$ led us to speculate that

13 naturally-occurring human variants might similarly dysregulate SARM1 and thereby increase

14 disease risk.

16 To investigate whether SARM1 mutations that disrupt its autoinhibition are associated with

17 neurogenerative disorders, we sought to identify rare prodegenerative SARM1 missense variants

18 in human databases. ALS warrants particular attention because peripheral axon degeneration

19 accompanies and may precede motoneuron death during ALS progression ${ }^{19,20}$. Here, we identify

20 over two dozen such polymorphisms found in ALS patients and interrogate the activities of the

21 encoded enzymes in cultured neurons. Provocatively, the majority of our strongest candidate

22 variants disrupt SARM1 regulation and confer constitutive activity in vitro. Furthermore,

23 expression of a constitutively-active $S A R M 1$ allele found in three unrelated patients causes an 
1 ALS-like phenotype-motor dysfunction, axon loss and sustained neuroinflammation-when

2 expressed in the mouse spinal cord via intrathecal delivery.

3 We identified a total of 29 SARM1 coding variants (missense and small in-frame deletions)

4 culled from three large publicly-accessible ALS consortia databases that include 8,507 cases in

5 total (Table 1$)^{21-23}$.

6

7 Table 1 Rare SARM1 missense variants and in-frame deletions found in ALS patients

8

$\begin{array}{lll}\text { Variant } \quad \text { Percent Minor Allele Frequencya } & \begin{array}{l}\text { Number of } \\ \text { occurrences in ALS } \\ \text { patients }\end{array}\end{array}$

\begin{tabular}{llllll}
\multicolumn{2}{c}{} & African & $\begin{array}{l}\text { East } \\
\text { Asian }\end{array}$ & European & \\
\hline \multicolumn{2}{l}{ Constitutively active } & & & & \\
\hline$\Delta 226-232$ & rs782325355 & 0 & 0 & 0.01 & $2^{\mathrm{d}}$ \\
$\Delta 249-252$ & & 0 & 0 & 0 & $1^{\mathrm{d}}$ \\
V184G & rs71373646 & 0.007 & 0.006 & 0 & $3^{\mathrm{e}}$ \\
G206S & rs1555585199 & 0 & 0 & 0 & $2^{\mathrm{e}}$ \\
L23P & & 0 & 0 & 0 & $1^{\mathrm{d}}$ \\
R267W & rs11652384 & 0 & 0 & 0.001 & $1^{\mathrm{e}}$ \\
V331E & rs1555585331 & 0 & 0 & 0 & $1^{\mathrm{d}}$ \\
E340K & rs781854217 & 0 & 0 & 0.003 & $1^{\mathrm{d}}$ \\
T385A & & 0 & 0 & 0 & $1^{\mathrm{d}}$ \\
T502P & rs782421919 & 0 & 0 & 0.006 & $2^{\mathrm{d}, \mathrm{f}}$ \\
\hline
\end{tabular}

Not constitutively active

\begin{tabular}{llllll}
\hline A240E & rs1449836804 & 0 & 0 & 0.004 & $1^{d}$ \\
R244S & & 0 & 0 & 0 & $1^{d}$ \\
A250T & rs1555585243 & 0 & 0.06 & 0 & $1^{d}$ \\
A275V & rs376587698 & 0 & 0 & 0.006 & $1^{d}$
\end{tabular}

$\mathrm{R} 310 \mathrm{H} \quad \mathrm{rs369186722}$

A341V rs373458416

R403P rs782706244

E431G rs1555585662

R465T

R484C

A488E

V518L

R569C

R570Q

D637Y

A646S

M672V

S684F

rs1555585809

rs782228906

rs782106973

rs571724138

rs539229444

rs1451417529

rs782676389

rs782774927

rs782256561

R702C

rs781850558

0

0

$0.01 \quad 1 d$

9

10 agnomAD v2

11 bnon-Finnish

12 cV518L $=0.009 \%$ in gnomAD v3 non-Finnish Europeans

$13 \mathrm{dM}$ inE 32

14 eALSVariant Server 34

15 fALS Knowledge Portal ${ }^{33}$ 
1 Altogether, rare SARM1 variants (i.e. with allele frequencies $<0.01 \%$ in all gnomAD

2 populations $^{24}$ ) occur in $>0.9 \%$ of ALS cases but in only $\sim 0.25 \%$ of controls ${ }^{21,22}$, and in only

$3 \sim 0.3 \%$ of the general population ${ }^{24}$. For comparison, potentially pathogenic $T B K 1$ variants were

4 reported in $1.1 \%$ of ALS cases and $0.19 \%$ of controls ${ }^{25}$. This suggests that rare pathogenic

5 SARM1 alleles might contribute to ALS risk.

7 To investigate whether these variants disrupt autoinhibition, we assayed the $\mathrm{NAD}^{+}$hydrolase

8 activities of the encoded enzymes. We prioritized the variants and first tested a) those identified

9 in multiple ALS patients but not in healthy controls and b) those unique to ALS patients (i.e. not

10 reported in any prior human study as of January 2020). These 15 SARMI variants (Figure 1A,

11 Table 1) account for 51\% (20/39) of rare SARM1 variants in ALS patients genotyped in the three

12 large ALS databases we investigated. To examine the properties of these mutants, we tested them

13 in $\mathrm{Sarm}^{-/}$mouse dorsal root ganglion DRG neurons. We prepared lentiviruses for the 15

14 SARM1 mutant constructs, infected Sarm1 ${ }^{-/}$neurons, and assessed their NAD ${ }^{+}$hydrolase

15 activity. Eight of these variants were determined to be constitutively active, i.e. we found that the

16 baseline level of $\mathrm{NAD}^{+}$was decreased in neurons expressing these mutant constructs and the

17 level of cADPR, a specific in vitro and in vivo SARM1 biomarker ${ }^{26}$ was increased (Fig. 1). By

18 contrast, $S A R M 1^{\mathrm{P} 332 \mathrm{Q}}$, the only variant common in any gnomAD population (1.1\% in Europeans)

19 is not constitutively active (Fig 1).

21 Encouraged by these results, we assayed the activities of an additional 14 rare missense variants.

22 These were considered poorer candidates because each is observed in only a single ALS patient

23 and they are not unique to the patients as they are also found in the gnomAD database. Among 
1 these, we identified two additional constitutively active variants (Table 1). In total, 40\% (4/10) of

2 the SARM1 variants with constitutive $\mathrm{NAD}^{+}$hydrolase activity occur in multiple ALS patients.

4 Point mutants that disrupt SARM1's autoinhibitory interfaces result in dysregulation of SARM1

5 activity and promote the degeneration of cultured neurons ${ }^{14-18}$. Consistent with those findings,

6 lentiviral-mediated expression of all the constitutively active variants we tested (Table 1) alters

7 the cell body morphology of cultured $\mathrm{Sarm}^{-/}$mouse DRG neurons consistent with cell death.

8 To quantify this pro-degenerative activity, two variant constructs, $S A R M 1^{V 184 G}$ and $S A R M 1^{4226-}$

$9 \quad{ }^{232}$, were studied further. The mutant enzymes were expressed in Sarm ${ }^{-/}$DRGs neurons and

10 degeneration was measured by two methods. Fluorescently-labeled Annexin V, which binds to

11 phosphatidylserine, was used to determine whether the expression of either variant construct

12 significantly compromises axon health. Annexin $\mathrm{V}$ binding is a useful proxy for axon health as

13 neurites undergoing Wallerian degeneration expose phosphatidylserine on their extracellular

14 surfaces similarly to apoptotic cells ${ }^{27}$. Neuronal death was quantified using an oxidoreductase

15 activity assay, a common measure of cell viability. Both assays demonstrated that both ALS-

16 associated $S A R M 1$ variants produced a significant degenerative effect relative to the common

17 reference allele (Fig. 1).

19 While these variants exhibit constitutive $\mathrm{NAD}^{+}$hydrolase activity, it is formally possible that

20 they mediate their pro-degenerative effects via a distinct toxic mechanism. To investigate this

21 alternate hypothesis, we generated constructs containing two mutations, either of the ALS-

22 associated variants, $S A R M 1^{V 184 G}$ or $S A R M 1^{4226-232}$, together with E642A, a point mutation in the

23 TIR domain that disrupts the catalytic glutamate required for SARM1 NAD ${ }^{+}$hydrolase activity 
1 and axon degeneration ${ }^{3}$. In both cases, introducing E642A abolishes enzymatic activity and the

2 detrimental effects of the constructs on cell body and axon health (Fig. 1). Hence, these ALS

3 patient-derived SARM1 variants promote degeneration via loss of autoinhibition and resulting

4 constitutive $\mathrm{NAD}^{+}$hydrolase activity.

6 To test whether the rare SARM1 variants promote neurodegeneration in vivo, AAV viral vectors

7 were administered intrathecally to male and female six-week old wild-type mice, expressing

8 either the common human allele of SARM1 (the reference allele) or SARM1 $1^{V 184 G}$, the

9 constitutively active variant found most frequently in the ALS patient databases. In these

10 constructs, each SARM1 protein was fused to EGFP and expressed under the control of the

11 human synapsin promoter. The AAV viruses were produced with these constructs and a mixture

12 of PHP.S and PHP.eB serotype capsids (both derived from AAV9 ${ }^{28}$ ) in order to infect neurons in

13 the spinal cord and DRGs.

15 Animals injected with AAV expressing the common SARMI allele had no discernible behavioral 16 phenotypes for at least 12 weeks. By contrast, those injected with AAV-SARMI $1^{V 184 G}$ exhibited

17 motor impairment. Two of the mice rapidly progressed to full limb paralysis 3-4 days after 18 injection. Other animals injected with $S A R M 1^{V 184 G}(7 / 9)$ displayed less dramatic motor deficits

19 characterized by hindlimb clasping ${ }^{29}$, and significant muscle weakness, as measured by the

20 inverted screen assay (Fig. 2). These deficits were detected within 3 weeks of injection and did 21 not progress significantly through the 12-week observation period. 
1 To characterize the neurodegeneration caused by $S A R M^{V 184 G}$ expression, the intrathecally

2 injected mice were examined for evidence of axon degeneration and neuron loss. We examined

3 the two mice that became rapidly paralyzed and the other mice with less severe disease as

4 separate cohorts because of the difference in phenotype. In the spinal cords of the paralyzed

5 mice, there was clear evidence of cell death around the ependymal canal as detected by TUNEL

6 staining. Neuroinflammation was observed throughout the spinal cord of these mice as evidenced

7 by prevalent staining for CD68, a marker of activated macrophages. Neither of these phenotypes

8 were observed in animals injected with the common SARM1 allele construct (Additional File 1).

9 These mice did not display obvious myelin defects or vacuolization in the sural, sciatic or tibial

10 nerves at 3-4 days post-infection.

12 The mice treated with $S A R M 1^{V 184 G}$ that displayed a less severe behavioral response were

13 sacrificed twelve weeks post-injection. Pathological inspection of their spinal cords revealed no

14 evidence of ongoing apoptosis or elevated CD68 staining. Their peripheral nerves, however,

15 contain almost 10-fold more CD68-positive macrophages than those treated with the control

$16 S A R M 1$ allele (Fig. 2). Macrophages also increase in size upon activation ${ }^{30}$, and the $S A R M 1^{V 184 G}$

17 infected mice have a 1.6-fold greater CD68-stained area per cell than do control mice, yielding a

18 15.2-fold difference in total CD68-stained area. Hence, neuronal expression of SARM1

19 triggers an elevated inflammatory response in peripheral nerves that persists for at least twelve

20 weeks after treatment ${ }^{31}$.

22 The average fiber densities in the peripheral nerves of the $S A R M 1^{V 184 G}$-injected mice are also

23 lower than those injected with AAV expressing the common human allele, demonstrating that 
1 expression of the constitutively active SARM1 promotes axon loss. The number of axons were

2 counted in the sural, sciatic and tibial nerves. In both the sural and sciatic nerves, the density of

3 axons is significantly lower $(\mathrm{p}<0.001)$, a trend that is evident in the tibial nerve, though it did not

4 reach statistical significance (Fig. 2). The average axon size and extent of myelination (g-ratio)

5 does not differ significantly between the variant and common SARM1 allele treated animals

$6(\mathrm{p}>0.1, \mathrm{n}=15)$. Myelin defects and vacuolization are not observed in these nerves, indicating a

7 lack of ongoing axon loss. The lack of axon defects at twelve weeks is consistent with the early,

8 but stable, deficits in motor function observed in mice receiving the SARM1 ${ }^{V 184 G}$ virus (Fig. 2).

9 We interpret these data as evidence that a subset of neurons- those sufficiently susceptible to

10 SARM1-dependent degeneration and infected with virus-lost their axons before three weeks,

11 while others, including uninfected neurons, remained healthy and functional up to twelve weeks.

12 Inter-animal differences in motor dysfunction severity likely reflect variability in infection

13 efficiency.

15 In summary, we find that many rare SARM1 variants found in ALS patients also lack normal

16 autoinhibition, and that such an allele induces neurodegeneration and neuroinflammation when

17 expressed in the mouse nervous system. We therefore propose that hypermorphic SARM1

18 mutations are a candidate congenital risk factor for ALS. The mechanism by which constitutive

$19 \mathrm{NAD}^{+}$hydrolase activity would predispose to neurodegeneration appears straightforward as low

$20 \mathrm{NAD}^{+}$is a death sentence for energy-hungry neurons and is associated with both disease and

21 aging-related functional defects ${ }^{32}$. We speculate that the contrast between virus-transfected mice

22 that rapidly display severe degenerative phenotypes, and human ALS patients who are typically

23 diagnosed only after several decades of life, likely reflects the difference in SARM1 
1 expression-i.e. viral over-expression precipitates abrupt metabolic catastrophe in this model,

2 whereas chronic suboptimal $\mathrm{NAD}^{+}$levels lead to gradual motoneuron attrition in patients.

3 Fortunately, small molecule SARM1 inhibitors are already in development ${ }^{33}$, and we have shown

4 that a SARM1 dominant negative gene therapy can potently block the SARM1 programmed axon

5 degeneration pathway in mice ${ }^{34}$. Establishing that SARM1 inhibition is safe and effective in

6 carriers of pathogenic SARM1 variants could provide a vital stepping stone toward the use of

7 SARM1-directed therapeutics more generally for ALS and other diseases that involve axon

8 degeneration.

\section{Methods}

\section{Mice}

14 Male and female WT and Sarm1 knockout C57/BL6 mice were housed and used under the

15 direction of institutional animal study guidelines at Washington University in St. Louis. The

16 inverted screen test of strength was performed as previously ${ }^{35}$. All protocols received

17 institutional IACUC approval.

\section{DRG culture}

20 Mouse DRG culture was performed as previously described ${ }^{36}$. DRG were dissected from

21 embryonic day 13.5 Sarml knockout C57/BL6 mouse embryos and cells suspended in growth

22 medium at a concentration of $\sim 7 \times 10^{6}$ cells $/ \mathrm{ml}$ in $96-$ well tissue culture plates (Corning) coated

23 with poly-d-Lysine $(0.1 \mathrm{mg} / \mathrm{ml}$; Sigma $)$ and laminin $(3 \mu \mathrm{g} / \mathrm{ml}$; Invitrogen $)$. For axotomy, 
$1 \quad$ suspended neurons $(2 \mu \mathrm{l})$ were placed as a drop in the center of each well and incubated at $37^{\circ} \mathrm{C}$

2 with $5 \% \mathrm{CO}_{2}$ for $15 \mathrm{~min}$, after which media was added to each well. Lentiviral particles

3 containing SARM1 variants were generated as previously described ${ }^{36}$. Lentivirus was added (1-

$410 \times 10^{3} \mathrm{pfu}$ ) after 1-2 days (DIV) and metabolites were extracted or assays were performed at

5 6-7 DIV. Cell death was quantified by assaying mitochondrial function (MTT assay), as

6 previously described ${ }^{37}$.

\section{Automated quantification of axon degeneration}

9 The axon degeneration index, the ratio of fragmented axon area over total axon area, was

10 quantified as previously described ${ }^{36}$. To quantify Annexin V staining, the Alexa Fluor ${ }^{\mathrm{TM}} 568$

11 conjugate (ThermoFisher) was added to the cultured neurons at a 1:100 dilution four days after

12 viral infection. Bright field and fluorescent images were acquired one hour later using

13 Operetta. Unbiassed image analysis was performed using ImageJ as follows: total axon area was

14 measured from the binary bright field images after subtracting background. For Annexin

15 fluorescent intensity measurement, the fluorescent images were background subtracted and then

16 annexin positive area was defined using the particle analyzer. Data was reported as the total

17 fluorescent intensity of the annexin positive area divided by the axon area.

\section{DRG metabolite extraction and metabolite measurement}

20 At DIV6, tissue culture plates were placed on ice and culture medium replaced with ice-cold

21 saline $(0.9 \% \mathrm{NaCl}$ in water, $500 \mu \mathrm{l}$ per well). Saline was removed and replaced with $160 \mu \mathrm{l}$ ice

22 cold $50 \% \mathrm{MeOH}$ in water. Solution was transferred to tubes containing $50 \mu$ chloroform on ice,

23 shaken vigorously, and centrifuged at $20,000 \mathrm{~g}$ for $15 \mathrm{~min}$ at $4{ }^{\circ} \mathrm{C}$. The clear aqueous phase 
1 (140 $\mu \mathrm{l})$ was transferred into microfuge tubes and lyophilized under vacuum. Lyophilized

2 samples were reconstituted with $5 \mathrm{mM}$ ammonium formate $(15 \mu \mathrm{l})$, centrifuged $(13,000 \mathrm{~g}, 10$

$3 \mathrm{~min}, 4^{\circ} \mathrm{C}$ ), and $10 \mu \mathrm{l}$ of clear supernatant was analyzed. $\mathrm{NAD}^{+}$and $\mathrm{cADPR}$ were measured using

$4 \quad$ LC-MS/MS as previously described ${ }^{26}$.

6 AAV constructs and virus injections

7 AAV particles with a mixture of Php.s and Php.eB capsids ${ }^{28}$, containing a human SARM1 gene

8 construct fused to EGFP, under the control of the human synapsin promoter, were produced by

9 the Viral Vector Core of the Hope Center for Neurological Disorders at Washington University

10 in St. Louis. Viral particles were purified by iodixanol gradient ultracentrifugation and virus

11 titers were measured by dot blot. Under light anesthesia with Avertin, $6 \times 10^{11}$ viral genomes

12 were injected intrathecally at L6/S1. Viral expression in mice 12-weeks post injection was

13 confirmed by detecting EGFP expression via immunohistochemical analysis of sectioned DRGs.

\section{Immunohistochemistry, imaging and quantification}

16 After perfusion with PBS followed by $4 \%$ PFA in PBS, tissues were fixed in $4 \%$ PFA in PBS for

$171 \mathrm{~h}$ at room temperature and placed in $30 \%$ sucrose in PBS overnight at $4^{\circ} \mathrm{C}$, then embedded in

18 OCT (Tissue-Tek), frozen on dry ice, and then stored at $-80^{\circ} \mathrm{C}$. Longitudinal sections of $6 \mu \mathrm{m}$ or

19 cross-sections of $20 \mu \mathrm{m}$ were obtained using a cryostat and slides were stored at $-20^{\circ} \mathrm{C}$. DRG

20 and nerve slides were post-fixed in cold acetone, then washed with PBS. Spinal cord slides were

21 simply washed three times in PBS. All slides were subsequently blocked with 4\% BSA and $1 \%$

22 Triton X-100 in PBS and incubated with rat anti-CD68 (1:500; Bio-Rad) and mouse-anti-GFP

23 conjugated to Alexa Fluor 488 (1:250; Thermo Fisher Scientific) overnight in the blocking 
1 buffer. Slides were then washed, incubated in secondary antibodies (Jackson ImmunoResearch

2 Laboratories) and mouse anti-GFP conjugated to AlexaFlour 488 (1:250, Thermo Fisher

3 Scientific), washed, and mounted in Vectashield with DAPI. Slides were imaged using a DMI

4 4000B confocal microscope (Leica Microsystems) with a 20× oil objective and DFC 7000-T

5 camera (Leica Microsystems). For quantification, at least four images were measured per animal.

6 CD68-positive cells were counted by a researcher blinded to the images' treatment group. The

7 total CD68-stained area and nerve area in each image was quantified with the particle analyzer in

8 ImageJ using a uniform threshold.

\section{TUNEL apoptosis detection}

11 TUNEL was performed as previously described ${ }^{38}$. Slides prepared for immunohistochemistry

12 were thawed then postfixed with $4 \%$ PFA for $10 \mathrm{~min}$ at room temperature, washed thoroughly

13 with $\mathrm{PBS}$, incubated with $10 \mu \mathrm{g} / \mathrm{ml}$ proteinase $\mathrm{K}$ for $15 \mathrm{~min}$ at $37^{\circ} \mathrm{C}$, then washed with $\mathrm{PBS}$. A

14 positive control slide was incubated in DNase I $(1 \mathrm{U} / \mathrm{ml})$ for $1 \mathrm{~h}$ at $\mathrm{RT}$, then washed with PBS.

15 Slides were then pretreated with TdT buffer $(25 \mathrm{~mm}$ Tris- $\mathrm{HCl}, 200 \mathrm{~mm}$ sodium cacodylate, 0.25

$16 \mathrm{mg} / \mathrm{ml} \mathrm{BSA}, 1 \mathrm{~mm}$ cobalt chloride, Roche Diagnostics) at RT for $10 \mathrm{~min}$. To perform end-

17 labeling, TdT buffer was combined with terminal deoxynucleotidyl transferase (Roche

18 Diagnostics, 400 U/slide) and Biotin-16-dUTP (Roche Diagnostics, $4 \mu \mathrm{m}$ ) and added to slides

19 for $1 \mathrm{~h}$ at $37^{\circ} \mathrm{C}$. Slides were thoroughly washed with PBS, then blocked for 30 min with 5\%

20 normal goat serum in PBS with $0.3 \%$ Triton-X, then incubated with Alexa-Fluor-conjugated

21 streptavidin (Jackson ImmunoResearch Laboratories) for $30 \mathrm{~min}$ at $37^{\circ} \mathrm{C}$. Slides were washed

22 and mounted in Vectashield with DAPI. 


\section{Toluidine blue staining and axon quantification}

2 Sural, sciatic and tibial nerves were fixed in $3 \%$ glutaraldehyde in $0.1 \mathrm{M}$ PBS, processed and

3 imaged as previously described ${ }^{8}$. Micrographs were stitched using Leica software and axons

4 were counted using ImageJ. To determine axon size distribution and G ratios of the sciatic nerve,

5 four nonoverlapping areas per cross section were imaged with a $100 \times$ oil objective of a Zeiss

6 Axioskop and photographed with a Hitachi camera. Photographs were analyzed using a

7 previously described binary imaging analysis method ${ }^{39}$.

$9 \quad$ Statistical analysis

10 Two-tailed significance is reported throughout. All statistics were calculated using the R

11 software package. All data is available upon request.

\section{Abbreviations}

15 AAV: Adeno-associated virus

16 ALS: Amyotrophic Lateral Sclerosis

17 ARM: HEAT/Armadillo motif

18 cADPR: Cyclic adenosine diphosphate ribose

19 CD68: Cluster of Differentiation 68

20 DRG: Dorsal root ganglion

21 EGFP: Enhanced green fluorescent protein

22 MLS: Mitochondrial localization signal

23 MTT: 3-(4,5-dimethylthiazol-2-yl)-2,5-diphenyltetrazolium bromide 
1 NAD: Nicotinamide adenine dinucleotide

2 NMNAT2: Nicotinamide mononucleotide adenylyltransferase 2

3 TIR: Toll/Interleukin receptor

4 TUNEL: Terminal deoxynucleotidyl transferase dUTP nick end labeling

5 SAM: Sterile alpha motif

6 SARM1: Sterile alpha and TIR motif containing 1

\section{Declarations}

\section{Ethical Approval and Consent to participate}

10 All studies were approved by the Washington University Institutional Animal Care and Use

11 Committee.

\section{Consent for publication}

13 Not Applicable

\section{Availability of supporting data}

15 All data relevant to this study are contained within the article.

\section{Competing interests}

17 A.D. and J.M. are co-founders, scientific advisory board members and shareholders of Disarm

18 Therapeutics, a wholly owned subsidiary of Eli Lilly. A.J.B. and Y.S. are consultants to Disarm

19 Therapeutics. The authors have no other competing conflicts or financial interests.

\section{Funding}

21 This work was supported by National Institutes of Health grants (R01NS119812 to A.J.B., A.D.

22 and J.M, R01NS087632 to A.D. and J.M., R37NS065053 to A.D., and RF1AG013730 to J.M.)

\section{Authors' contributions}


1 A.J.B, J.M and A.D designed the study. X.M performed in vitro experiments. A.S

2 performed in vivo experiments. Y.S assisted with mass spec analysis and method

3 development. A.J.B. analyzed the data. A.J.B., J.M. and A.D. drafted and edited the

4 figures and manuscript. All authors read and approved the final manuscript.

\section{Acknowledgements}

6 We extend our thanks to Tim Fahrner and Kelli Simburger for cloning viral constructs, to Rachel

7 McClarney and Cassidy Menendez for assistance with mouse husbandry and administering

8 behavioral tests, and to Alicia Neiner for processing LC-MS/MS samples. This work was

9 supported by the Hope Center Viral Vectors Core at Washington University School of Medicine.

\section{Authors' information}

11 Not Applicable

\section{References}

17 1. Figley MD, DiAntonio A. The SARM1 axon degeneration pathway: control of the NAD+ 18 metabolome regulates axon survival in health and disease. Curr Opin Neurobiol. 2020.

19 doi:10.1016/j.conb.2020.02.012

20 2. Gilley J, Ribchester RR, Coleman MP. Sarm1 Deletion, but Not WldS, Confers Lifelong

$21 \quad$ Rescue in a Mouse Model of Severe Axonopathy. Cell Rep. 2017.

22 doi:10.1016/j.celrep.2017.09.027

23 3. Essuman K, Summers DW, Sasaki Y, Mao X, DiAntonio A, Milbrandt J. The SARM1 
Toll/Interleukin-1 Receptor Domain Possesses Intrinsic NAD+ Cleavage Activity that Promotes Pathological Axonal Degeneration. Neuron. 2017. doi:10.1016/j.neuron.2017.02.022

4. Gerdts J, Brace EJ, Sasaki Y, DiAntonio A, Milbrandt J. SARM1 activation triggers axon degeneration locally via NAD ${ }^{+}$destruction. Science (80- ). 2015. doi: $10.1126 /$ science. 1258366

5. Gerdts J, Summers DW, Sasaki Y, DiAntonio A, Milbrandt J. Sarm1-mediated axon degeneration requires both SAM and TIR interactions. J Neurosci. 2013. doi:10.1523/JNEUROSCI.1197-13.2013

6. Osterloh JM, Yang J, Rooney TM, et al. dSarm/Sarm1 is required for activation of an injury-induced axon death pathway. Science (80- ). 2012. doi:10.1126/science.1223899

7. Ozaki E, Gibbons L, Neto NGB, et al. SARM1 deficiency promotes rod and cone photoreceptor cell survival in a model of retinal degeneration. Life Sci Alliance. 2020. doi:10.26508/lsa.201900618

8. Geisler S, Doan RA, Strickland A, Huang X, Milbrandt J, DiAntonio A. Prevention of vincristine-induced peripheral neuropathy by genetic deletion of SARM1 in mice. Brain. 2016. doi:10.1093/brain/aww251

9. Henninger N, Bouley J, Sikoglu EM, et al. Attenuated traumatic axonal injury and improved functional outcome after traumatic brain injury in mice lacking Sarm1. Brain. 2016. doi:10.1093/brain/aww001

10. Ko KW, Milbrandt J, DiAntonio A. SARM1 acts downstream of neuroinflammatory and necroptotic signaling to induce axon degeneration. J Cell Biol. 2020.

doi:10.1083/JCB.201912047 
1 11. Sasaki Y, Kakita H, Kubota S, et al. SARM1 depletion rescues NMNAT1-dependent

2 photoreceptor cell death and retinal degeneration. Elife. 2020. doi:10.7554/eLife.62027

3 12. Huppke P, Wegener E, Gilley J, et al. Homozygous NMNAT2 mutation in sisters with

$4 \quad$ polyneuropathy and erythromelalgia. Exp Neurol. 2019. doi:10.1016/j.expneurol.2019.112958

6 13. Gilley J, Mayer PR, Yu G, Coleman MP. Low levels of NMNAT2 compromise axon development and survival. Hum Mol Genet. 2019. doi:10.1093/hmg/ddy356

8 14. Figley MD, Gu W, Nanson JD, et al. SARM1 is a metabolic sensor activated by an

9 increased NMN/NAD+ ratio to trigger axon degeneration. Neuron. 2021.

10 doi:https://doi.org/10.1016/j.neuron.2021.02.009

11 15. Sporny M, Guez-Haddad J, Khazma T, et al. Structural basis for SARM1 inhibition and 12 activation under energetic stress. Elife. 2020. doi:10.7554/eLife.62021

13 16. Jiang Y, Liu T, Lee CH, Chang Q, Yang J, Zhang Z. The NAD+-mediated self-inhibition mechanism of pro-neurodegenerative Sarm1. Nature. 2020. doi:10.1038/s41586-0202862-Z

16 17. Shen C, Vohra M, Zhang P, et al. Multiple domain interfaces mediate SARM1 17 autoinhibition. Proc Natl Acad Sci. 2021. doi:10.1073/pnas.2023151118

18 18. Bratkowski M, Xie T, Thayer DA, et al. Structural and Mechanistic Regulation of the Prodegenerative NAD Hydrolase SARM1. Cell Rep. 2020. doi:10.1016/j.celrep.2020.107999

20 19. Fischer LR, Culver DG, Tennant P, et al. Amyotrophic lateral sclerosis is a distal 21 axonopathy: Evidence in mice and man. Exp Neurol. 2004. doi:10.1016/j.expneurol.2003.10.004

23 20. Clark JA, Southam KA, Blizzard CA, King AE, Dickson TC. Axonal degeneration, distal 
collateral branching and neuromuscular junction architecture alterations occur prior to symptom onset in the SOD1G93A mouse model of amyotrophic lateral sclerosis. J Chem Neuroanat. 2016. doi:10.1016/j.jchemneu.2016.03.003

21. van der Spek RAA, van Rheenen W, Pulit SL, Kenna KP, van den Berg LH, Veldink JH. The project MinE databrowser: bringing large-scale whole-genome sequencing in ALS to researchers and the public. Amyotroph Lateral Scler Front Degener. 2019. doi:10.1080/21678421.2019.1606244

22. Farhan SMK, Howrigan DP, Abbott L, et al. Enrichment of rare protein truncating variants in amyotrophic lateral sclerosis patients. bioRxiv. 2018. doi:10.1101/307835

23. Kenna KP, Van Doormaal PTC, Dekker AM, et al. NEK1 variants confer susceptibility to amyotrophic lateral sclerosis. Nat Genet. 2016. doi:10.1038/ng.3626

24. Karczewski KJ, Francioli LC, Tiao G, et al. Variation across 141,456 human exomes and genomes reveals the spectrum of loss-of-function intolerance across human protein-coding genes. bioRxiv. 2019. doi:10.1101/531210

25. Cirulli ET, Lasseigne BN, Petrovski S, et al. Exome sequencing in amyotrophic lateral sclerosis identifies risk genes and pathways. Science (80- ). 2015. doi:10.1126/science.aaa3650

26. Sasaki Y, Engber TM, Hughes RO, et al. cADPR is a gene dosage-sensitive biomarker of SARM1 activity in healthy, compromised, and degenerating axons. Exp Neurol. 2020. doi:10.1016/j.expneurol.2020.113252

27. Sievers C, Platt N, Perry VH, Coleman MP, Conforti L. Neurites undergoing Wallerian degeneration show an apoptotic-like process with annexin $\mathrm{V}$ positive staining and loss of mitochondrial membrane potential. Neurosci Res. 2003. doi:10.1016/S0168- 
2 28. Chan KY, Jang MJ, Yoo BB, et al. Engineered AAVs for efficient noninvasive gene delivery to the central and peripheral nervous systems. Nat Neurosci. 2017. doi:10.1038/nn.4593

29. Igaz LM, Kwong LK, Lee EB, et al. Dysregulation of the ALS-associated gene TDP-43

30. Ying W, Cheruku PS, Bazer FW, Safe SH, Zhou B. Investigation of macrophage polarization using bone marrow derived macrophages. J Vis Exp. 2013. doi: $10.3791 / 50323$

11 31. Mueller M, Wacker K, Ringelstein EB, Hickey WF, Imai Y, Kiefer R. Rapid response of identified resident endoneurial macrophages to nerve injury. Am J Pathol. 2001.

14 32. Covarrubias AJ, Perrone R, Grozio A, Verdin E. NAD+ metabolism and its roles in cellular processes during ageing. Nat Rev Mol Cell Biol. 2021. doi:10.1038/s41580-020-

33. Hughes RO, Bosanac T, Mao X, et al. Small Molecule SARM1 Inhibitors Recapitulate the SARM1-/- Phenotype and Allow Recovery of a Metastable Pool of Axons Fated to Degenerate. Cell Rep. 2021. doi:10.1016/j.celrep.2020.108588

34. Geisler S, Huang SX, Strickland A, et al. Gene therapy targeting SARM1 blocks pathological axon degeneration in mice. J Exp Med. 2019. doi:10.1084/jem.20181040

22 35. McGill BE, Barve RA, Maloney SE, et al. Abnormal microglia and enhanced inflammation-related gene transcription in mice with conditional deletion of Ctcf in 
Camk2a-Cre-expressing neurons. J Neurosci. 2018. doi:10.1523/JNEUROSCI.0936-

17.2017

3 36. Sasaki Y, Vohra BPS, Lund FE, Milbrandt J. Nicotinamide mononucleotide adenylyl

4 transferase-mediated axonal protection requires enzymatic activity but not increased levels

5 of neuronal nicotinamide adenine dinucleotide. J Neurosci. 2009.

7 37. Mao X, Moerman AM, Lucas MM, Barger SW. Inhibition of the activity of a neuronal «B-binding factor by glutamate. J Neurochem. 1999. doi:10.1046/j.1471-

10 38. Sasaki Y, Hackett AR, Kim S, Strickland A, Milbrandt J. Dysregulation of NAD+

11 metabolism induces a Schwann cell dedifferentiation program. J Neurosci. 2018. doi:10.1523/JNEUROSCI.3304-17.2018

13 39. Hunter DA, Moradzadeh A, Whitlock EL, et al. Binary imaging analysis for

14 comprehensive quantitative histomorphometry of peripheral nerve. J Neurosci Methods. 


\section{$1 \quad$ Figure Legends}

3 Figure 1. Dysregulated SARM1 variants found in ALS patients promote

neurodegeneration. (A) Schematic representation of the domain structure of SARM1.

5 Constitutively-active variants are indicated above in red. Bold variants were prioritized because they were identified in multiple ALS patients or were unique to ALS patients. $\Delta$ indicates an in-

7 frame deletion. MLS, mitochondrial localization signal; ARM, HEAT/Armadillo motif; SAM,

8 sterile alpha motif; TIR, Toll/interleukin-1 receptor homology domain. (B) cADPR and NAD ${ }^{+}$

9 levels from cultured Sarm $1^{-/-}$DRG neurons infected with variant human SARM1 constructs,

10 performed in triplicate, relative to the reference $S A R M 1$ allele. (C) Neuron death as measured by

11 the MTT assay and (D) axon degeneration as measured by Annexin V staining in Sarm $1^{-/-}$DRG

12 neurons infected with lentivirus expressing $S A R M 1$ variant constructs as well as double mutant

13 constructs including E642A, a point mutation that disrupts SARM1 NAD ${ }^{+}$hydrolase activity,

14 relative to the common $S A R M 1$ reference allele, all performed in triplicate. (E) Representative

15 bright-field and Annexin V-stained images of axons from Sarm $1^{-/-}$DRG cultures infected with

16 variant and SARM1 reference allele constructs. $* \mathrm{p}<0.05 ; * * \mathrm{p}<0.005 ; * * * \mathrm{p}<0.0005$ difference

17 from reference allele, two-tailed t-test.

19 Figure 2. Motor dysfunction, neuroinflammation and axon loss in mice injected

20 intrathecally with a SARM1 ${ }^{V 184 G}$ AAV construct. (A) Average time suspended from an

21 inverted screen (maximum 120 seconds, performed in triplicate) for C57/BL6 mice injected with

22 a human $S A R M 1$ reference allele $(\mathrm{n}=8)$ or $S A R M 1^{V 184 G}(\mathrm{n}=7)$ AAV compared to uninjected

23 controls $(n=3)$ 3, 9 and 12 weeks post-injection. ${ }^{*} p<0.005$ difference from both the reference 
1 allele and uninjected controls, 2-tailed t-test. (B) The normalized average number of cells stained

2 by the macrophage marker anti-CD68 in nerve, and the average percent area of total anti-CD68

3 staining in nerve and in spinal cord sections, from C57/BL6 mice injected with a SARM1 ${ }^{V 184 G}$

4 AAV construct (3 images per mouse, $\mathrm{n}=7$ mice) relative to those injected with a human SARM1

5 reference allele construct ( $\mathrm{n}=8$ mice) 12 weeks post-injection; $* \mathrm{p}<10^{-4}, 2$-tailed $\mathrm{t}$-test. (C)

6 Representative images of nerve stained with DAPI and anti-CD68 from mice 12 weeks after

7 injection with a $S A R M 1^{V 184 G}$ or reference allele construct. (D) Average fibers per cross-sectional

$8 \mu \mathrm{m}^{2}$ in sural, sciatic and tibial nerves from mice 12 weeks after injection with a $S A R M 1^{V 184 G}(3$

9 images per mouse, $\mathrm{n}=7$ mice) or reference allele construct $(\mathrm{n}=8$ mice $) ;{ }^{*} \mathrm{p}<0.05 ; * * \mathrm{p}<0.001,2$ -

10 tailed t-test. (E) Representative images of toluidine blue stained sural nerve sections.

12 Additional file 1. Rapid cell death and neuroinflammation in mice injected intrathecally

13 with a SARM1 $1^{V 184 G}$ AAV construct.

14 (A) Representative images of spinal cord sections, with closeup of ependymal canal, stained with

15 DAPI and the apoptosis marker TUNEL from mice 2 days after injection with a $S A R M 1^{V 184 G}$ or

16 SARM1 human reference allele construct. (B) Representative images of spinal cord and (C)

17 adjacent nerve sections stained with DAPI and the macrophage marker anti-CD68 from mice 2

18 days after injection with a $S A R M 1^{V 184 G}$ or reference allele construct. 
bioRxiv preprint doi: https://doi.org/10.1101/2021.04.16.439886; this version posted April 16, 2021. The copyright holder for this preprint (which Figure 1 as not certified by peer review) is the author/funder, who has granted bioRxiv a license to display the preprint in perpetuity. It is made
available under aCC-BY-NC 4.0 International license.

A

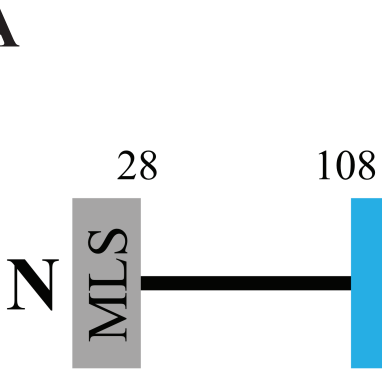

กิ กิ กิ

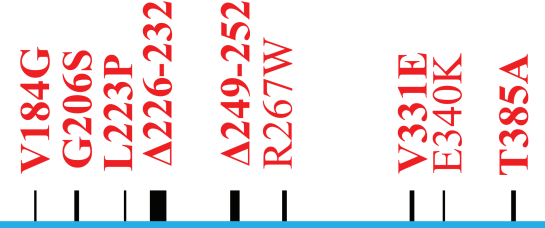

ARM

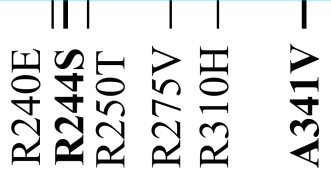

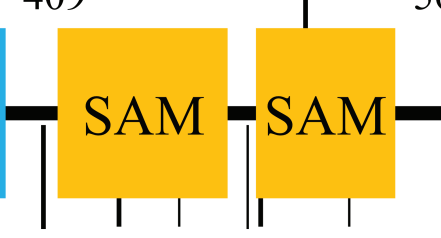

563

724

\section{B}

\section{cADPR}

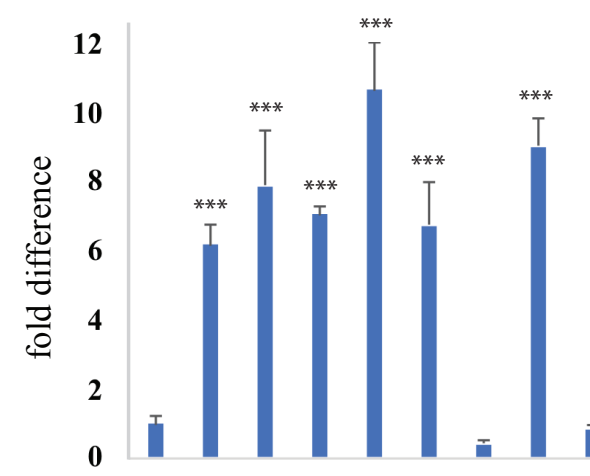
$5 \sin ^{20}$

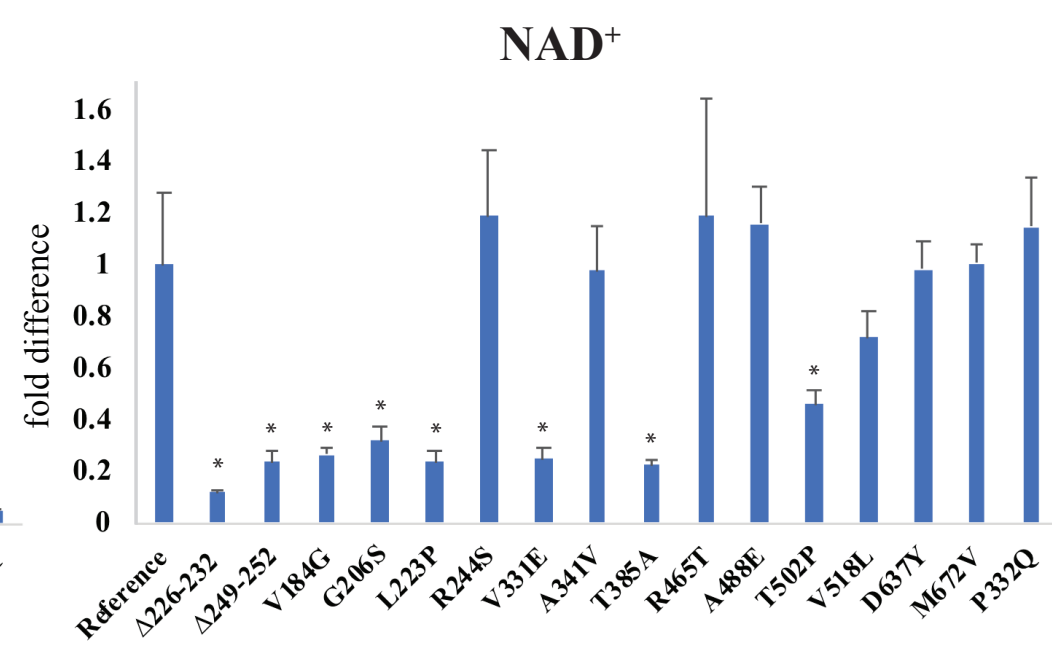

$\mathbf{E}$

Reference

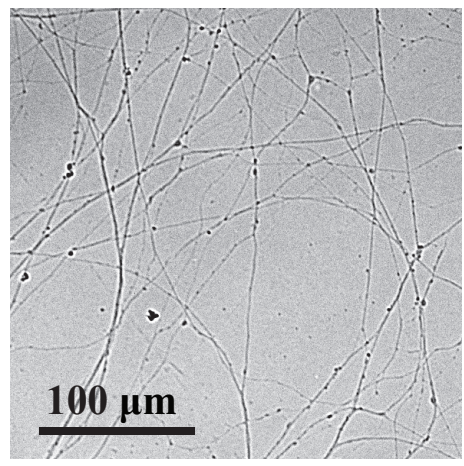

(1)

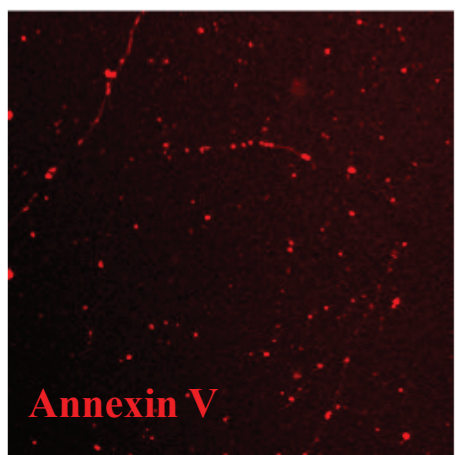

V184G
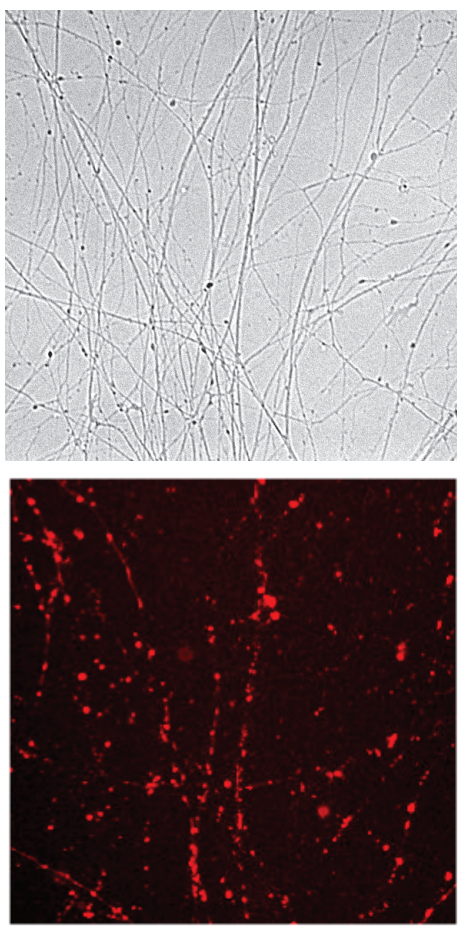

V184G; E642A
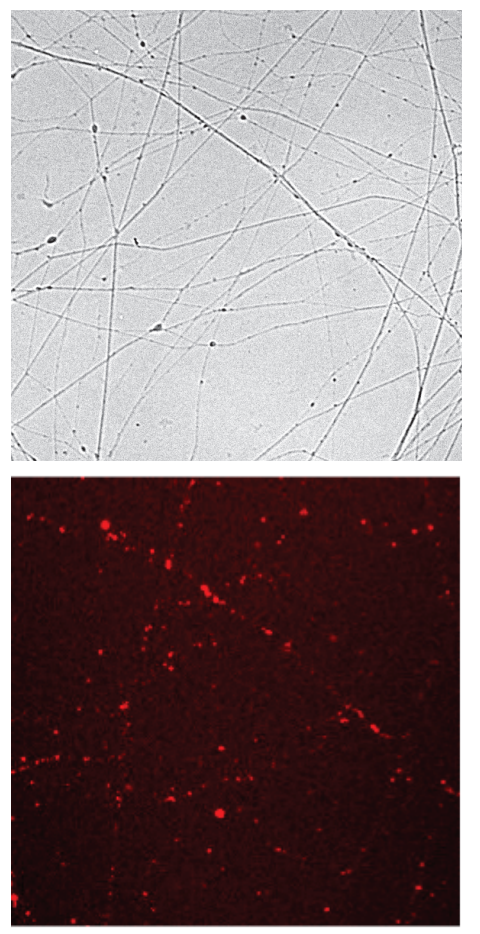
bioRxiv preprint doi: https://doi.org/10.1101/2021.04.16.439886; this version posted April 16, 2021. The copyright holder for this preprint (which Figure 2 wast certified by peer review) is the author/funder, who has granted bioRxiv a license to display the preprint in perpetuity. It is made

A
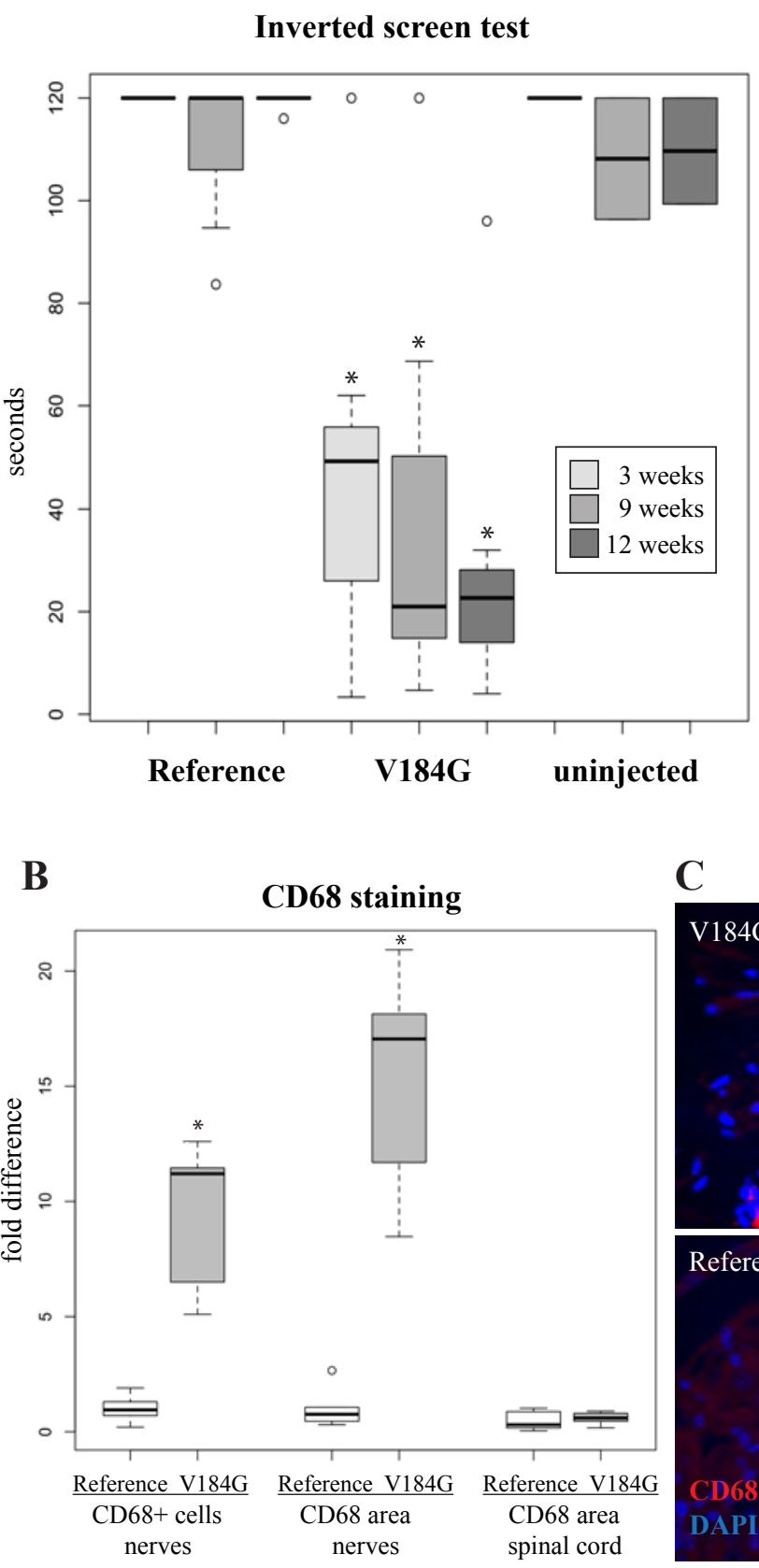

C

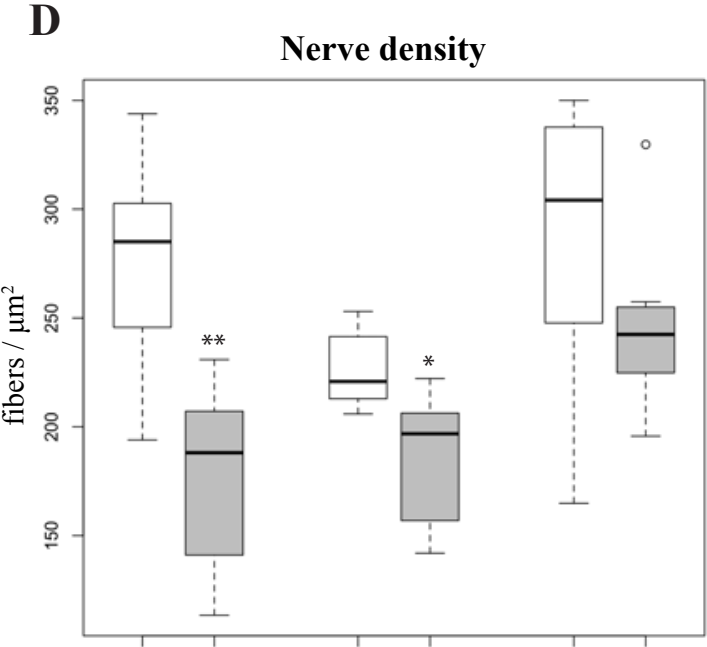

Reference V184G Reference V184G Reference V184G sural sciatic tibial
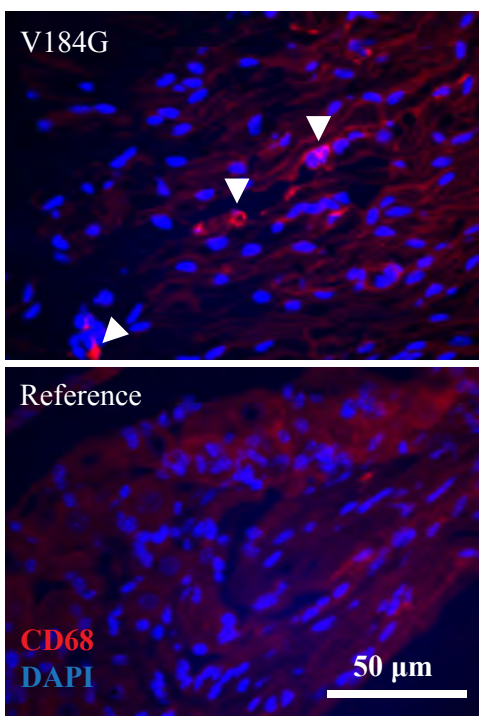

$\mathbf{E}$

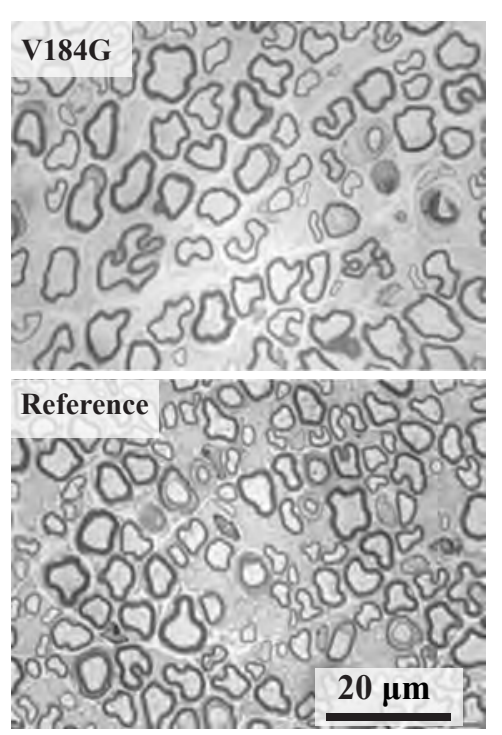


Additional File 1

A

\section{V184G}

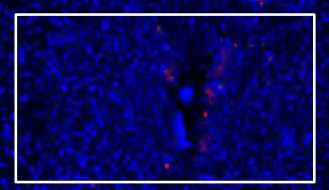

TUNEL

DAPI

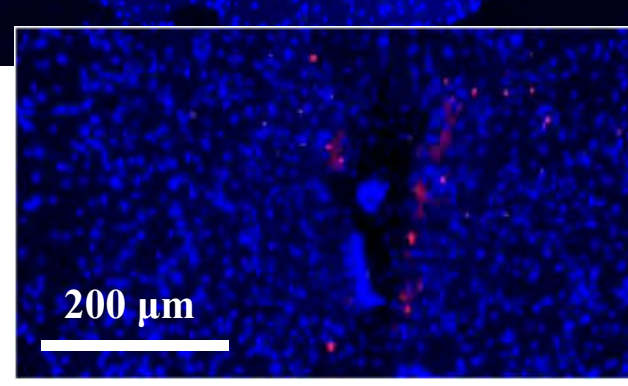

B

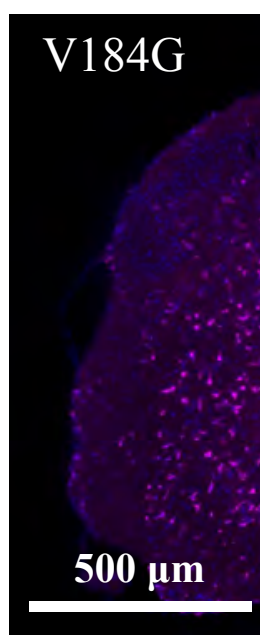

CD68

DAPI
Reference
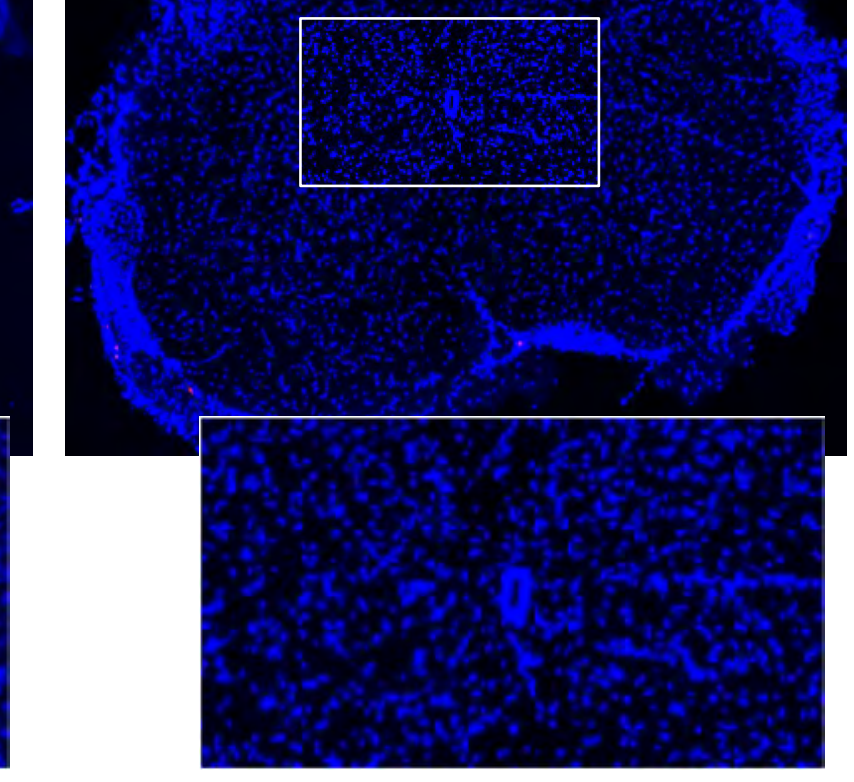

C

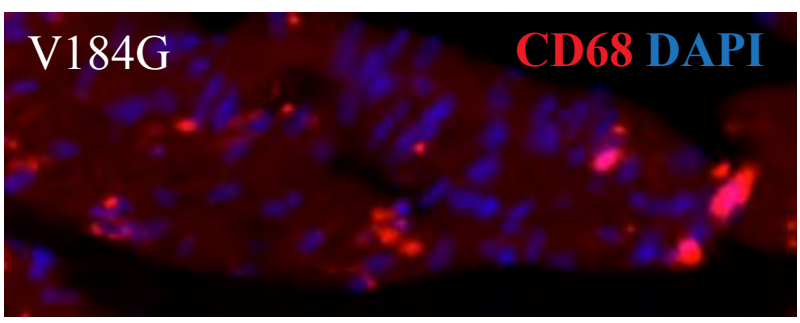

Reference

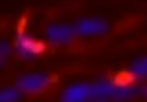

$50 \mu \mathrm{m}$ 